Edition KWV 
Die „Edition KWV“ beinhaltet hochwertige Werke aus dem Bereich der Wirtschaftswissenschaften. Alle Werke in der Reihe erschienen ursprünglich im Kölner Wissenschaftsverlag, dessen Programm Springer Gabler 2018 übernommen hat.

Weitere Bände in der Reihe http://www.springer.com/series/16033 
Sandra Wagner

\section{Entscheidungsorientiertes Umweltkostenmanagement}

Konzeption zur aktiven Gestaltung von Umweltkosten im betrieblichen Umweltmanagement

Springer Gabler 
Sandra Wagner

MARGA Business Simulations GmbH

Erftstadt, Deutschland

Bis 2018 erschien der Titel im Kölner Wissenschaftsverlag, Köln

Dissertation Universität Siegen, 2010

\section{Edition KWV}

ISBN 978-3-658-24362-3 ISBN 978-3-658-24363-0 (eBook)

https://doi.org/10.1007/978-3-658-24363-0

Die Deutsche Nationalbibliothek verzeichnet diese Publikation in der Deutschen Nationalbibliografie; detaillierte bibliografische Daten sind im Internet über http://dnb.d-nb.de abrufbar.

\section{Springer Gabler}

(C) Springer Fachmedien Wiesbaden GmbH, ein Teil von Springer Nature 2010, Nachdruck 2019

Ursprünglich erschienen bei Kölner Wissenschaftsverlag, Köln, 2010

Das Werk einschließlich aller seiner Teile ist urheberrechtlich geschützt. Jede Verwertung, die nicht ausdrücklich vom Urheberrechtsgesetz zugelassen ist, bedarf der vorherigen Zustimmung des Verlags. Das gilt insbesondere für Vervielfältigungen, Bearbeitungen, Übersetzungen, Mikroverfilmungen und die Einspeicherung und Verarbeitung in elektronischen Systemen.

Die Wiedergabe von allgemein beschreibenden Bezeichnungen, Marken, Unternehmensnamen etc. in diesem Werk bedeutet nicht, dass diese frei durch jedermann benutzt werden dürfen. Die Berechtigung zur Benutzung unterliegt, auch ohne gesonderten Hinweis hierzu, den Regeln des Markenrechts. Die Rechte des jeweiligen Zeicheninhabers sind zu beachten.

Der Verlag, die Autoren und die Herausgeber gehen davon aus, dass die Angaben und Informationen in diesem Werk zum Zeitpunkt der Veröffentlichung vollständig und korrekt sind. Weder der Verlag, noch die Autoren oder die Herausgeber übernehmen, ausdrücklich oder implizit, Gewähr für den Inhalt des Werkes, etwaige Fehler oder Äußerungen. Der Verlag bleibt im Hinblick auf geografische Zuordnungen und Gebietsbezeichnungen in veröffentlichten Karten und Institutionsadressen neutral.

Springer Gabler ist ein Imprint der eingetragenen Gesellschaft Springer Fachmedien Wiesbaden GmbH und ist ein Teil von Springer Nature

Die Anschrift der Gesellschaft ist: Abraham-Lincoln-Str. 46, 65189 Wiesbaden, Germany 


\section{Geleitwort}

Der betriebliche Umweltschutz gewinnt heute mehr und mehr an wettbewerblicher Bedeutung. Dies gilt einerseits für die Erzielung von Kostenvorteilen gegenüber den Wettbewerbern und andererseits für die Verbesserung der marktlichen Position, zum Beispiel in der Umweltschutzindustrie, der Automobilindustrie, der Nahrungsmittelindustrie, der Textilindustrie usw. Diese Aufzählung lässt sich nahezu beliebig fortsetzen, wobei heute kaum noch ein Markt existiert, für den Umweltschutzaspekte ohne Bedeutung sind. Hinzu kommen neuere umweltpolitische Instrumente, zum Beispiel der Emissionshandel, die ebenfalls große Auswirkungen auf die wirtschaftliche Erfolgsposition eines Unternehmens haben können. Aus diesem Grund ist es nur folgerichtig, dass sich Unternehmen nach innen mit den Faktoren, Prozessen und Produkten beschäftigen, von denen negative Umweltauswirkungen ausgehen, und nach außen Rechenschaft gegenüber den betrieblichen Anspruchsgruppen ablegen. Umweltschutzaspekte spielen dabei in fast allen Entscheidungen eine wichtige Rolle. Bei Investitionsentscheidungen sind die von den zu Grunde liegenden Technologien ausgehenden Umweltwirkungen in ihrer wirtschaftlichen Tragweite zu beachten. Bei Entscheidungen über das Produktprogramm sind das Umweltbewusstsein der Kunden sowie sonstige marktliche Aspekte, die den betrieblichen Umweltschutz betreffen, einzubeziehen. Bei der Produktionsplanung muss sichergestellt werden, dass ordnungsrechtliche Vorgaben, zum Beispiel in Form von Emissionsgrenzwerten, möglichst kostenminimal eingehalten werden.

In dieser als Dissertationsschrift verfassten Monographie widmet sich Frau Dr. Sandra Wagner dem entscheidungsorientierten Umweltkostenmanagement und stellt eine neue Konzeption vor, die die aktive Gestaltung von Umweltkosten unterstützt. Dabei stellt sie einen „Werkzeugkasten“ bereit, der für verschiedene Entscheidungs- und sonstige Auswertungszwecke relevante Informationen liefert. Das vorgestellte Optimierungsmodell baut auf in Unternehmen vorhandenen Informationsstrukturen auf und ermöglicht es Unternehmen, die Folgewirkungen ihrer Entscheidungen für das Kostenniveau, die Kostenstruktur und den Kostenverlauf präzise abschätzen zu können. Das Modell enthält mit der Integration von Umweltabgaben, des Emissionshandels, der Möglichkeit des Recyclings und des Wiedereinsatzes von Ressourcen, von Emissionsgrenzwerten und der Begrenzung des Umweltschadens nahezu alle für die Produktionsplanung relevanten Aspekte zur Einbeziehung von betrieblichen Umweltwirkungen. Da es sich um ein lineares Optimierungsmodell handelt, lassen sich umweltbezogene Verrechnungspreise mithilfe von Schattenpreisen ermitteln, wobei zu berücksichtigen ist, dass es sich hier um keine Allokation pagatorischer Kosten, sondern vielmehr um Grenzpreise handelt. Das Modell stellt insgesamt Möglichkeiten der Einbeziehung von Umweltwirkungen in die Produktionsplanung schlüssig dar und baut sachlogisch gut auf den zuvor erarbeiteten Systematiken auf. 
Die von Frau Dr. Wagner entwickelte geschlossene Umweltkostenmanagementkonzeption ist darüber hinaus auch für die betriebliche Praxis von hoher Bedeutung, da sie alle umweltbezogenen Aspekte systematisch und sachgerecht verarbeiten kann. Die Arbeit liefert einen umfassenden Überblick zu Fragen des umweltorientierten Kostenmanagements und ist damit sowohl für Wissenschaftler als auch für Praktiker interessant. Die klare Modellstruktur, die präzise herausgearbeiteten Ergebnisse und schließlich die vorgestellten Methoden liefern eine sehr gute Grundlage für weitere Forschungsarbeiten und können der betrieblichen Praxis als Handlungsleitlinie zur Verbesserung des Kostenmanagements und zur Erzielung von umweltbezogenen Wettbewerbsvorteilen dienen.

Siegen / Boulder im Juli 2010

Augsburg im Juli 2010

Peter Letmathe

Günter Beuermann 


\section{Vorwort}

Mit den Themen Umweltkostenrechnung und Umweltkostenmanagement hatte ich mich bereits im Rahmen meines betriebswirtschaftlichen Studiums, insbesondere in der Diplomarbeit, intensiv auseinandergesetzt. Da in Teilaspekten mehr Fragen aufgeworfen als Antworten gegeben wurden, lag die Entscheidung nahe, dieses Forschungsgebiet weiter $\mathrm{zu}$ bearbeiten. Das entscheidungsorientierte Umweltkostenmanagement sollte mich in den folgenden Jahren beschäftigen und herausfordern. Dabei wurde schnell deutlich, dass die Verzahnung von Umweltkostenmanagement und Umweltmanagement insbesondere auf der Ebene der betrieblichen Gestaltungsbereiche vorliegt - also bezüglich der eingehenden Ressourcen (Input), der Transformationsprozesse (Throughput) und der erstellten erwünschten und nicht erwünschten Leistungen (Outputs). Die Parallelen zum Prozess der Erstellung der vorliegenden Dissertation liegen auf der Hand:

- Input in Form von Literatur, Konzepten, Laptop und Arbeitskraft - vor allem jedoch die vielen fachlichen und auch emotionalen Gespräche, die mir immer wieder frische Ideen und neue Motivation gegeben haben,

- Throughput in Form von Denken und Schreiben, Mut und auch Zweifeln, Pausen und durcharbeiteten Nächten - vor allem aber die oben genannten unerlässlichen Ideen und notwendige Motivation als ständig laufender Motor,

- Output natürlich in Form der vorliegenden Arbeit (und vielen verworfenen Ideen sowie zahlreichen Ordnern mit Notizen und Fachartikeln) - aber auch wertvollen Erfahrungen, neu erworbenen Fähigkeiten und die Kenntnis über eigene Stärken und Schwächen.

In dem gesamten Prozess haben mich viele Menschen begleitet und unermüdlich unterstützt:

Mein besonderer Dank gilt meinem ersten Doktorvater, Herrn Prof. Dr. Peter Letmathe, der mir und meinem Dissertationsthema im Jahr 2003 an seinem Lehrstuhl an der Universität Siegen ein neues Zuhause gegeben und mich stets kompetent unterstützt sowie nach Kräften gefordert und gefördert hat. Ein ebensolcher Dank geht an meinen zweiten Doktorvater, Herrn Prof. Dr. Dr. h.c. Günter Beuermann, der mich ursprünglich für das Projekt Promotion begeistert hat und an dessen Lehrstuhl an der Universität zu Köln ich zunächst als wissenschaftliche Mitarbeiterin tätig war. Seine inhaltliche Unterstützung und seine freundschaftliche Verbundenheit haben mich maßgeblich geleitet. Herrn Prof. Dr. Volker Stein danke ich herzlich für die Übernahme des Vorsitzes der Promotionskommission und die angenehmen Organisation und Durchführung des Rigorosums.

Allen Kolleginnen und Kollegen des Siegener Lehrstuhlkreises um Prof. Dr. Peter Letmathe bin ich zu großem Dank verpflichtet. Die kollegiale Auseinandersetzung im Rahmen der Dissertationserstellung war stets wertvoll und weiterführend. 
Mein besonderer Dank gilt dem Freundes- und Mitarbeiterkreis des Kölner Lehrstuhls, dem Team Beuermann. Herr Dr. Ralf Brüning, Herr Dr. Mahammad Mahammadzadeh, Herr Dr. Martin Kupp, Herr Dr. Frank Czymmek, Frau Dr. Ellen FaßbenderWynands, Herr Dr. Björn Maier, Frau Dr. Annette Boms sowie Dietlind Eicker waren mir in all den Jahren unersetzliche und geduldige Weggefährten, Mitstreiter und Kritiker.

Gerade in der Endphase der Promotionszeit haben mir meine Kolleginnen und Kollegen der MARGA Business Simulations GmbH in Erftstadt großzügige zeitliche Freiräume für die Fertigstellung der Dissertation und für die Vorbereitung auf das Rigorosum zugestanden. Auch ihnen gilt mein großer Dank.

Ablenkende Zerstreuung und aufmunternden Zuspruch habe ich stets in meinem Familien- und Freundeskreis gefunden - insbesondere bei meinen Zirkel-Damen Claudia Stern und Miriam Wigger sowie bei meinem Patenkind Michèle Hirschfeld und meiner Patentante Gerda Hirschfeld. Gleiches gilt für die gesamte Whitmer-Familie, die in den USA ein zweites Zuhause und damit einen unersetzlichen Rückzugsort für mich geschaffen haben. Ihnen allen danke ich für die tatkräftige Unterstützung und das Verständnis für all die Belastungen, die eine Promotion mit sich bringen kann.

Unermesslich ist der Beitrag, den meine Familie geleistet hat. Meine Eltern Annegret und Horst Wagner, mein Bruder Holger mit seiner Frau Regina und seinem Sohn Jonas, meine Schwester Marina mit ihrem Freund Basti und auch meine Oma Bieshausen haben durch ihre Liebe, ihren Glauben an meine Fähigkeiten und ihr Vertrauen in mich den wesentlichen Grundstein für den Erfolg dieses Vorhabens gelegt. Ihre unermüdliche Unterstützung und Bestätigung sowie die regelmäßigen Ablenkungs- aber auch Motivationsmanöver haben mich immer wieder ermutigt.

Gleiches gilt für meinen Lebensgefährten Hendrik Brecht. Den Rückhalt, den ich bei ihm finde, und die Geduld, die Launen einer im Promotionsstress stehenden Person zu ertragen, haben mir die Arbeit an der Dissertation sehr erleichtert. Die zeitlichen Entbehrungen, die er hinnehmen musste, hat er mit Fürsorge, Verständnis, Stolz und Liebe beantwortet.

Hendrik und meiner Familie ist diese Arbeit gewidmet.

Köln im August 2010

Sandra Wagner 
Inhaltsverzeichnis

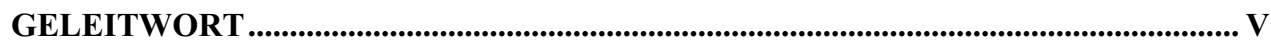

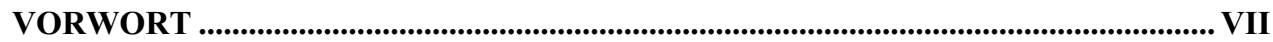

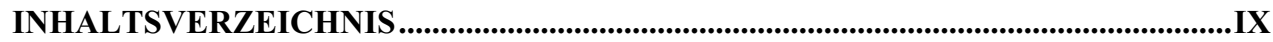

ABBILDUNGSVERZEICHNIS .............................................................................................III

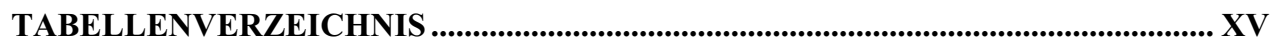

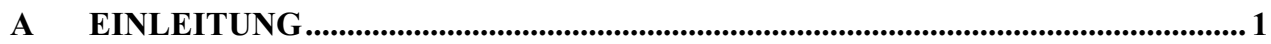

1 GEGENSTAND UND ZIELSETZUNG DER UNTERSUCHUNG......................... 1

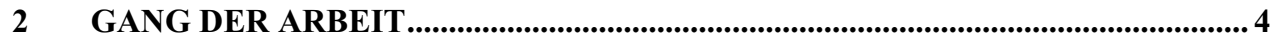

3 NOTWENDIGKEIT EINES UMWELTKOSTENMANAGEMENTS ..................... 6

3.1 Von der Kostenrechnung zum Kostenmanagement.......................................... 6

3.2 Veränderungen in der Unternehmensumwelt - Komplexität als

Kostentreiber ................................................................................................ 7

3.3 Auswirkungen auf die Kostensituation ........................................................ 11

3.4 Motive für die Einführung eines Umweltkostenmanagements ........................ 13

B ERKENNTNISOBJEKTE DER ARBEIT: KREISLAUFWIRTSCHAFT

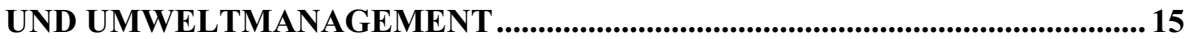

1 KREISLAUFWIRTSCHAFTLICHE BETRACHTUNG DES

UNTERNEHMENS ALS AUSGANGSSITUATION FÜR UMWELT-

MANAGEMENT UND UMWELTKOSTENMANAGEMENT

1.1 Gesamtwirtschaftliche Betrachtung kreislaufwirtschaftlicher

Zusammenhänge.

1.1.1 Entwicklung von der Durchlauf- zur Kreislaufwirtschaft - ein Paradigmenwechsel ............................................................................. 19

1.1.2 Zentrale Elemente eines allgemeinen Kreislaufwirtschaftsmodells .......... 24

1.1.3 Kritische Würdigung des Konzepts der Kreislaufwirtschaft .................... 29

1.2 Einzelwirtschaftliche Betrachtung kreislaufwirtschaftlicher Zusammenhänge: Implikationen für und Ansatzpunkte in Unternehmen......................... 30

1.2.1 Idealtypisches Modell einer betrieblichen Kreislaufwirtschaft ................. 30 
1.2.2 Die Kreislaufwirtschaft als betrieblicher Komplexitätstreiber.................. 36

1.2.3 Betriebliche Umweltwirkungen in der Kreislaufwirtschaft und deren Implikationen für Unternehmen

2 BETRIEBLICHES UMWELTMANAGEMENT IM RAHMEN DER

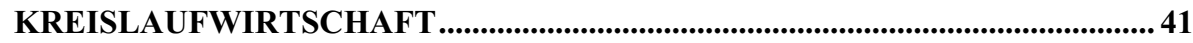

2.1 Grundlagen eines Umweltmanagements ......................................................44 44

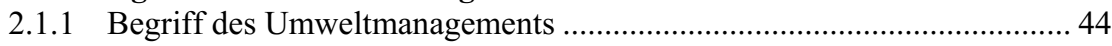

2.1.2 Ziele, Aufgaben und Anforderungen....................................................... 45

2.1.3 Potenzielle Chancen und Hemmnisse eines betrieblichen Umweltmanagements .................................................................. 48

2.1.4 Standardisierung des Umweltmanagements Umweltmanagementsysteme ................................................................ 51

2.2 Dimensionen des Umweltmanagements ..........................................................5 57

2.2.1 Ebenen des Umweltmanagements ...................................................... 57

2.2.1.1 Normatives Umweltmanagement ............................................ 58

2.2.1.2 Strategisches Umweltmanagement.......................................... 59

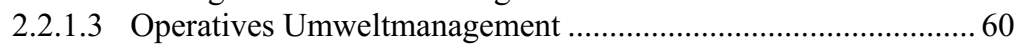

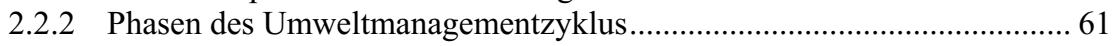

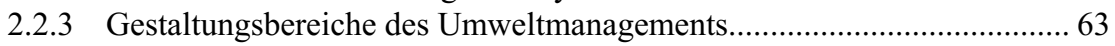

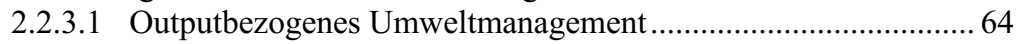

2.2.3.2 Inputbezogenes Umweltmanagement........................................66

2.2.3.3 Throughputbezogenes Umweltmanagement .............................. 67

2.3 Implikationen für das Umweltkostenmanagement ........................................69

C ENTSCHEIDUNGSUNTERSTÜTZENDES UMWELTKOSTEN-

MANAGEMENT IM BETRIEBLICHEN UMWELTMANAGEMENT ............... 71

1 GRUNDLAGEN DES BETRIEBLICHEN UMWELTKOSTEN-

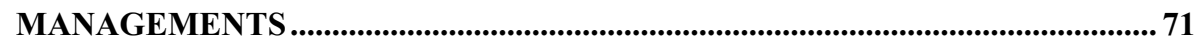

1.1 Umweltkosten als Zielgröße des Umweltkostenmanagements......................... 71

1.1.1 Definition und Umweltkostenbegriffe............................................... 72

1.1.2 Kosteneinflussgrößen im betrieblichen Umweltschutz .......................... 77

1.1.3 Bestimmung von Umweltkosten …................................................... 82

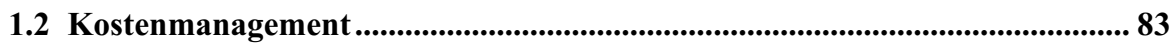

1.2.1 Begriffsdefinition ............................................................................. 83

1.2.2 Umweltkostenmanagement und Umweltkostenrechnung ....................... 84

2 OPTIMIERUNGSMODELL ZUR GESTALTUNG VON

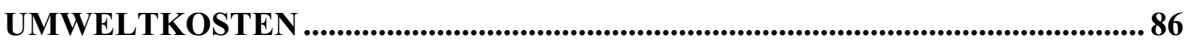

2.1 Überblick über Optimierungsansätze im Forschungsfeld

Umweltkostenmanagement.................................................................................. 86 
2.2 Modell zur Bestimmung der optimalen Prozesswahl unter

Berücksichtigung von Umweltkosten ....................................................................... 88

2.2.1 Problemdefinition und -formulierung..................................................... 90

2.2.2 Nummerisches Beispiel ..................................................................... 98

2.3 Ergebnisse des Optimierungsmodells.................................................................... 107

2.3.1 Minimalkostenkombination bei gegebenem Produktionsniveau............. 107

2.3.2 Interne Verrechnungspreise als entscheidungsrelevante Information ..... 109

3 MODELLGESTÜTZTE HERLEITUNG EINER ENTSCHEIDUNGSUNTERSTÜTZENDEN UMWELTKOSTENMANAGEMENT-

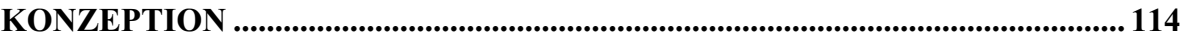

3.1 Umweltkostenmanagement und Umweltmanagement................................... 114

3.2 Dimensionen des Umweltkostenmanagements .................................................. 120

3.2.1 Kostenmerkmale im Umweltkostenmanagement................................ 121

3.2.2 Ausrichtung des Umweltkostenmanagements..................................... 124

3.2.3 Gestaltungsbereiche des Umweltkostenmanagements .......................... 125

3.2.4 Zusammenführung der drei Dimensionen des Umweltkostenmanagements ......................................................... 127

3.3 Handlungsfelder des Umweltkostenmanagements....................................... 128

3.3.1 Throughputbezogenes Umweltkostenmanagement.............................. 128

3.3.1.1 Gestaltung throughputbezogener Entscheidungsobjekte........... 128

3.3.1.2 Implikationen des Optimierungsmodells für throughputbezogene Entscheidungen...................................... 129

3.3.2 Inputbezogenes Umweltkostenmanagement ........................................ 131

3.3.2.1 Gestaltung inputbezogener Entscheidungsobjekte..................... 131

3.3.2.2 Implikationen des Optimierungsmodells für inputbezogene Entscheidungen ............................................. 132

3.3.3 Outputbezogenes Umweltkostenmanagement.................................... 134

3.3.3.1 Gestaltung outputbezogener Entscheidungsobjekte.................. 134

3.3.3.2 Implikationen des Optimierungsmodells für outputbezogene Entscheidungen ............................................. 135

\section{UMWELTKOSTENMANAGEMENT ALS} ENTSCHEIDUNGSUNTERSTÜTZENDES INSTRUMENTARIUM ................. 138

4.1 Zielhierarchie des Umweltkostenmanagements ............................................... 138

4.2 Rechnungszwecke eines entscheidungsunterstützenden

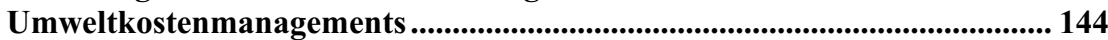

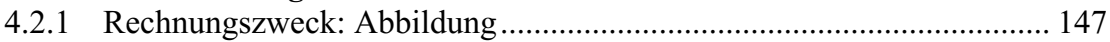

4.2.2 Rechnungszweck: Planung ........................................................... 150

4.2.3 Rechnungszweck: Steuerung und Kontrolle ....................................... 152

4.3 Entscheidungsorientierter Einsatz der Umweltkostenmanagement-

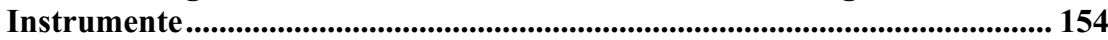

4.3.1 Auswahl der relevanten Umweltkostenmanagement-Instrumente.......... 154 
4.3.2 Auswertungszweckanalyse der UmweltkostenmanagementInstrumente

4.3.3 Auswertungszweckbezogene Einsatzpotenziale der Umweltkostenmanagement-Instrumente.

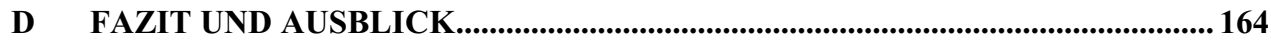

ANHANG 1: FORMULIERUNG DES NUMMERISCHEN BEISPIELS IN GAMS... 166

ANHANG 2: ERGEBNISSE DES NUMMERISCHEN BEISPIELS........................... 173

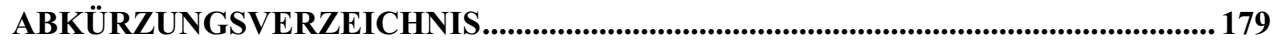

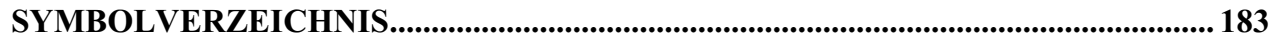

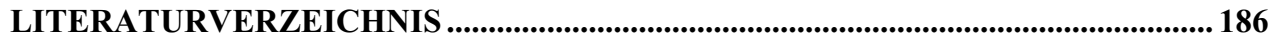

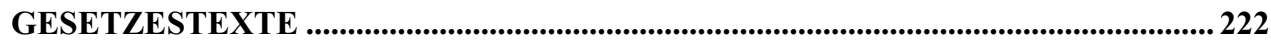




\section{Abbildungsverzeichnis}

Abb. A-1: Veränderungen in der Unternehmensumwelt

Abb. A-2: Gesamtwirtschaftliche Tendenzen und ihre Auswirkungen im Unternehmen

Abb. B-1: Vereinfachtes Kreislaufwirtschaftsmodell unter Berücksichtigung der Schnittstellen zwischen Ökosphäre und Technosphäre

Abb. B-2: Einzelwirtschaftliches Modell einer Kreislaufwirtschaft. 33

Abb. B-3: Externe und interne Vorteile und Hemmnisse des betrieblichen Umweltmanagements

Abb. B-4: Zykluselemente der ISO 14001 54

Abb. C-1: Systematik der Umweltkosten 74

Abb. C-2: Kosteneinflussgrößensystem des Umweltkostenmanagements. 81

Abb. C-3: Minimalkostenfunktion bei optimaler Prozesswahl 107

Abb. C-4: Schema zur Ermittlung der internen Verrechnungspreise der Inputfaktoren 110

Abb. C-5: Interne Verrechnungspreise der primären Inputfaktoren im nummerischen Beispiel 110

Abb. C-6: Schema zur Ermittlung der internen Verrechnungspreise der Emissionen

Abb. C-7: Interne Verrechnungspreise der Produktionsemissionen im nummerischen Beispiel

Abb. C-8: Interne Verrechnungskosten je erstellter Produkteinheit im nummerischen Beispiel

Abb. C-9: Integration von Umweltmanagement und Umweltkostenmanagement..... 114

Abb. C-10: Auftrag des Umweltkostenmanagements

Abb. C-11: Umweltkostenmanagement-Zyklus

Abb. C-12: Herleitung des entscheidungsunterstützenden

Umweltkostenmanagements. 
Abb. C-14: Durchführungshäufigkeit der Produktionsprozesse im nummerischen Beispiel

Abb. C-15: Durchführungshäufigkeit der Recyclingprozesse im nummerischen Beispiel....

Abb. C-16: Einsatzmenge primärer Inputfaktoren im nummerischen Beispiel 133

Abb. C-17: Einsatzmenge sekundärer Inputfaktoren im nummerischen Beispiel...... 134

Abb. C-18: Emissionsmenge aus der Produktion im nummerischen Beispiel 136

Abb. C-19: Menge zugekaufter Emissionszertifikate im nummerischen Beispiel..... 137

Abb. C-20: Zusammenhang von Umweltkostenstruktur, -verlauf und -höhe 140

Abb. C-21: Zielordnung des Umweltkostenmanagements..... 141

Abb. C-22: Umweltkostenverläufe 143

Abb. C-23: Morphologischer Kasten zur Typologie von betrieblichen umweltbezogenen Entscheidungssituationen

Abb. C-24: Kategorisierung der Rechnungszwecke im Umweltkostenmanagement

Abb. C-25: Rechnungsziele in der Kategorie Abbildung \& Dokumentation 149

Abb. C-26: Entscheidungsbaum zur auswertungszweckorientierten Instrumentenwahl 162 


\section{Tabellenverzeichnis}

Tab. B-1: Überblick über verschiedene umweltbezogene Strategietypologien.

Tab. C-1: Klassische Kosteneinflussgrößensysteme 78

Tab. C-2: Differenz aus im Recycling aufgenommenen und im Recycling generierten Emissionsmengen 94

Tab. C-3: Inputparameter. 99

Tab. C-4: Maximale Ressourcenverfügbarkeit der spezifischen Inputfaktoren für die Produktionsprozesse 100

Tab. C-5: Maximale Ressourcenverfügbarkeit der spezifischen Inputfaktoren für die Recyclingprozesse 100

Tab. C-6: Emissionsparameter. 101

Tab. C-7: Parameter der Umweltschadenskategorie 101

Tab. C-8: Input- bzw. Ressourcenkoeffizienten 102

Tab. C-9: Produktionsprozesskoeffizienten 103

Tab. C-10: Recyclingprozesskoeffizienten 105

Tab. C-11: Produktkoeffizienten 106

Tab. C-12: Umweltschadenskoeffizient 107

Tab. C-13: Fragestellungen zu den Kostenmerkmalen. 123

Tab. C-14: Fragestellungen zur Ausrichtung. 125

Tab. C-15: Fragestellungen zu den Gestaltungsbereichen 126

Tab. C-16: Kategorien der Umweltkostenrechnungsansätze 156

Tab. C-17: Ausgewählte Instrumente des Umweltkostenmanagements und deren Auseinandersetzung in der Fachliteratur. 
Tab. C-18: Rechnungszwecke der

Umweltkostenmanagement-Instrumente

Tab. C-19: Ausrichtung der

Umweltkostenmanagement-Instrumente

Tab. C-20: Auswertungszwecke der

Umweltkostenmanagement-Instrumente 160

Tab. Anhang-1: Interne Verrechnungspreise je Emissions- bzw.

Inputfaktoreinheit

Tab. Anhang-2: Interne Verrechnungspreise je Produkteinheit und Minimalkosten bei gegebenem Produktionsniveau

Tab. Anhang-3: Benötigte Einsatzmengen der primären und sekundären Inputfaktoren

Tab. Anhang-4: Emissionsmengen und Anzahl der zugekauften

Emissionsrechte

Tab. Anhang-5: Durchführungshäufigkeit der Produktionsprozesse

Tab. Anhang-6: Durchführungshäufigkeit der Recyclingprozesse 178 